\title{
A far infrared view of low mass star formation in the Cederblad 110 nebula of Chamaeleon $I^{\star}$
}

\author{
K. Lehtinen ${ }^{1}$, L. K. Haikala ${ }^{1,2}$, K. Mattila ${ }^{1}$, and D. Lemke ${ }^{3}$ \\ 1 Observatory, Tähtitorninmäki, PO Box 14, 00014 University of Helsinki, Finland \\ 2 Swedish-ESO Submillimetre Telescope, European Southern Observatory, Casilla 19001, Santiago, Chile \\ 3 Max-Planck-Institut für Astronomie, Königstuhl 17, 69117 Heidelberg, Germany
}

Received 28 June 2000 / Accepted 22 November 2000

\begin{abstract}
The Cederblad 110 reflection nebula contains several young stellar objects previously discovered by IRAS and sub-mm observations. This cluster was revisited with ISO's FIR cameras and mapped with higher spatial resolution. All sources were also mapped for the first time near the maximum of their spectral energy distribution at $200 \mu \mathrm{m}$. In addition, two new FIR-sources were discovered. The new data enabled parameters of the young objects to be determined, such as their luminosity, temperature and mass of the circumstellar dust. These parameters were related to the YSO classes of the various objects ranging from the pure protostar Class $\mathrm{O}$ to Class III. Cederblad 110 can be viewed as a compact volume of active low mass star formation in a dense ridge of molecular gas of the Chamaeleon I cloud detected e.g. in $\mathrm{HN}^{13} \mathrm{C}$ and $\mathrm{C}^{18} \mathrm{O}$ lines. The star formation efficiency in the $0.001 \mathrm{pc}^{3}$ cluster volume is high; about $20 \%$ of the gas is now bound into stars.
\end{abstract}

Key words. stars: formation - ISM: clouds - ISM: individual objects: Cederblad 110, IRAS 11048-7706, IRAS 11051-7706, IRAS 11057-7706

\section{Introduction}

The Chamaeleon complex contains dark clouds (Chamaeleon I, II and III) located at $b \sim-15^{\circ}$. The Chamaeleon I and II clouds have many signs of recent low-mass star formation (for a review see e.g. Schwartz 1991). We have adopted a distance of $150 \mathrm{pc}$ for Chamaeleon I (Knude \& Høg 1998).

At optical wavelengths the Chamaeleon I molecular cloud is characterized by three conspicuous reflection nebulae which are designated as Cederblad 110, 111 and 112 (Cederblad 1946). The reflection nebula Cederblad 110 is illuminated by an early G-type T Tauri star, with IRAS identification 11048-7706.

For the densest part of the reflection nebula near the illuminating star, there are three entries in the IRAS Point Source Catalog. However, this area is confused in the standard IRAS catalogues, and thus the positional and flux density information is uncertain. By using the IRAS Chopped Photometric Channel observations at 50 and $100 \mu \mathrm{m}$, Prusti et al. (1991) were able to resolve three

Send offprint requests to: K. Lehtinen,

e-mail: kimmo.lehtinen@helsinki.fi

* Based on observations with ISO, an ESA project with instruments funded by ESA Member States (especially the PI countries: France, Germany, The Netherlands and the UK) and with the participation of ISAS and NASA. sources and to derive positional and flux density information. They were able to extract flux densities for these three sources at 12,25 and $60 \mu \mathrm{m}$ by utilizing suitably oriented scans from the raw survey data. In the nomenclature of Prusti et al. (1991) these three sources are called IRS 2, IRS 4 and IRS 6.

The spectral energy distributions (SEDs) of most known YSOs can be classified into three morphological classes forming an evolutionary sequence (see e.g. Lada \& Wilking 1984; Adams et al. 1987; Lada 1987; Wilking et al. 1989): Class I (deeply embedded YSOs with disks and massive circumstellar gas and dust envelopes), Class II (classical T Tauri stars) and Class III sources (weak-line $\mathrm{T}$ Tauri stars). Since this classification, a number of objects have been discovered which show SEDs that are well characterized by a single modified blackbody function. These sources are called extreme Class I sources or Class 0 sources, and they may be true protostars.

IRS 2 is the illuminating star of the reflection nebula, and also the strongest X-ray source in Chamaeleon I. It has an estimated bolometric luminosity of $3.6 L_{\odot}$ and, based on the near-IR spectral index, it was classified as a Class II source (Prusti et al. 1991). Based on the equivalent $\mathrm{H} \alpha$ width Henning et al. (1993) have classified it as a Class III source, i.e. a weak-line T Tauri star. Gürtler et al. (1999) have measured a spectrum of IRS 2 with the ISOPHOT-S spectrophotometer. The mid-IR 5.8-11.6 $\mu \mathrm{m}$ 
spectrum is dominated by emission features attributed to PAHs.

The sources IRS 4 and IRS 6 have estimated bolometric luminosities of 1.4 and $1.0 L_{\odot}$, respectively. Based on the near-IR spectral index they have both been classified as Class I sources, i.e. deeply embedded objects (Prusti et al. 1991). IRS 4 and IRS 6 are the only Class I sources in Chamaeleon I with spectral energy distributions that continue to rise above $60 \mu \mathrm{m}$.

Mattila et al. (1989) and Prusti et al. (1991) have mapped a bipolar CO molecular outflow around the triplet IRS 2, 4 and 6. Reipurth et al. (1996) have made a continuum mapping survey at $1300 \mu \mathrm{m}$ around the triplet IRS 2, 4 and 6 . About $76^{\prime \prime}$ south of IRS 2 and 4 they detected an object, Cha-MMS1, which is not pointlike in their beam of $\mathrm{HPBW} \approx 22^{\prime \prime}$, but may consist of three sources. They derived an integrated flux density of $948 \mathrm{mJy}$ for the triplet. Cha-MMS1 is located close to the center of the CO outflow found by Mattila et al. (1989) and is thus a good candidate for being its driving source. Reipurth et al. (1996) concluded that Cha-MMS1 is probably the driving source of the Herbig-Haro objects HH 49 and 50. They proposed that the object may be a protostar or a protostellar clump.

The SEDs of IRS 2, 4 and 6 have been observed over a wide wavelength range (see Prusti et al. 1991). However, the observations have so far not covered the maximum of the SED which is located beyond $100 \mu \mathrm{m}$. Using the ISOPHOT data at four wavelengths between $80-200 \mu \mathrm{m}$, we have observed the maximum of the far-IR dust emission. The better spatial sampling and higher sensitivity as compared with IRAS also enable us to detect new FIR point sources not seen by IRAS.

\section{Observations and data reduction}

The observations were made with the ISOPHOT instrument (Lemke et al. 1996) aboard the ISO (Kessler et al. 1996) satellite. We used the $\mathrm{C} 100$ and C200 detectors at reference wavelengths $80,100,150$ and $200 \mu \mathrm{m}$. The $80 \mu \mathrm{m}$ observations were made on August 14th, while the other observations were made on August 4th 1996 using PHT22, i.e. a raster map on a two-dimensional regular grid. The TDT numbers of the $80,100,150$ and $200 \mu \mathrm{m}$ observations are 27101201, 26101402, 26101501 and 26101401, respectively. The grid spacings in in-scan and cross-scan directions at 80 and $100 \mu \mathrm{m}$ were $135^{\prime \prime}$ and $135^{\prime \prime}, 135^{\prime \prime}$ and $90^{\prime \prime}$, respectively. At 150 and $200 \mu \mathrm{m}$ grid spacing was $180^{\prime \prime}$ at both directions.

The data analysis was conducted using $\mathrm{PIA}^{1}$ (ISOPHOT Interactive Analysis) V 8.0 (Gabriel et al. 1997). The detector ramps were corrected for nonlinearity, glitches in ramps were removed by using the

\footnotetext{
1 The ISOPHOT data presented in this paper were reduced using PIA, which is a joint development by the ESA Astrophysics Division and the ISOPHOT Consortium with the collaboration of the Infrared Processing and Analysis Center (IPAC). Contributing ISOPHOT Consortium institutes are DIAS, RAL, AIP, MPIK, and MPIA.
}

two-threshold glitch recognition method. The ramps were fitted with 1st order polynomials, and orbital position dependent dark currents were subtracted.

For the flat-field correction we have applied a statistical method: we correlate the value of the reference pixel at each raster position with other pixels. Instead of comparing the reference pixel only with pixels located at the same raster position, we take a mean of two particular pixels located symmetrically around the reference pixel. In this way, it is possible to reduce the scatter in the pixelto-pixel relation caused by a surface brightness gradient in the image.

The primary intensity calibrators are the fine calibration sources (FCSs), which bracket the actual map measurements. In the case of C100 camera the detectors suffer under response transients during the relatively short time of FCS measurement, that is they do not reach their final intensity levels. We have corrected for this effect by modelling the signal transients at the SRD level with the PIA signal drift interface, using offset exponential as the fitting function. In the case of C200 camera there is no such transient behaviour.

The flux density calibration at $100 \mu \mathrm{m}$ has been compared with the flux densities from the IRAS Point Source Catalog for several point sources located on a smooth background away from the Cederblad 110 region. The flux densities agree to within about $20 \%$.

\section{Results}

A colour map of the Chamaeleon I region is shown in Fig. 1, together with a Digitized Sky Survey ${ }^{2} B$-band image of the same region. The sources IRS 2, 4, 6 and Cha-MMS1, located near the reflection nebula, are marked with dots.

\subsection{New infrared sources IRS 10 and IRS 11}

Inspection of the maps shows that at all four wavelengths the point sources are located on the top of an extended ( $\sim 5$ arcmin) ridge-like emission which is roughly centered at IRS 4. IRS 2, 4 and 6 are resolved as separate sources at $80 \mu \mathrm{m}$, while at $100 \mu \mathrm{m}$ only IRS 4 and 6 can be separated. In the 80 and $100 \mu \mathrm{m}$ maps there is also an indication of a fourth source located south of IRS 2 . In the 150 and $200 \mu \mathrm{m}$ maps, all the possible sources are blended

\footnotetext{
${ }^{2}$ Based on photographic data obtained using The UK Schmidt Telescope. The UK Schmidt Telescope was operated by the Royal Observatory Edinburgh, with funding from the UK Science and Engineering Research Council, until 1988 June, and thereafter by the Anglo-Australian Observatory. Original plate material is copyright (c)the Royal Observatory Edinburgh and the Anglo-Australian Observatory. The plates were processed into the present compressed digital form with their permission. The Digitized Sky Survey was produced at the Space Telescope Science Institute under US Government grant NAG W-2166.
} 


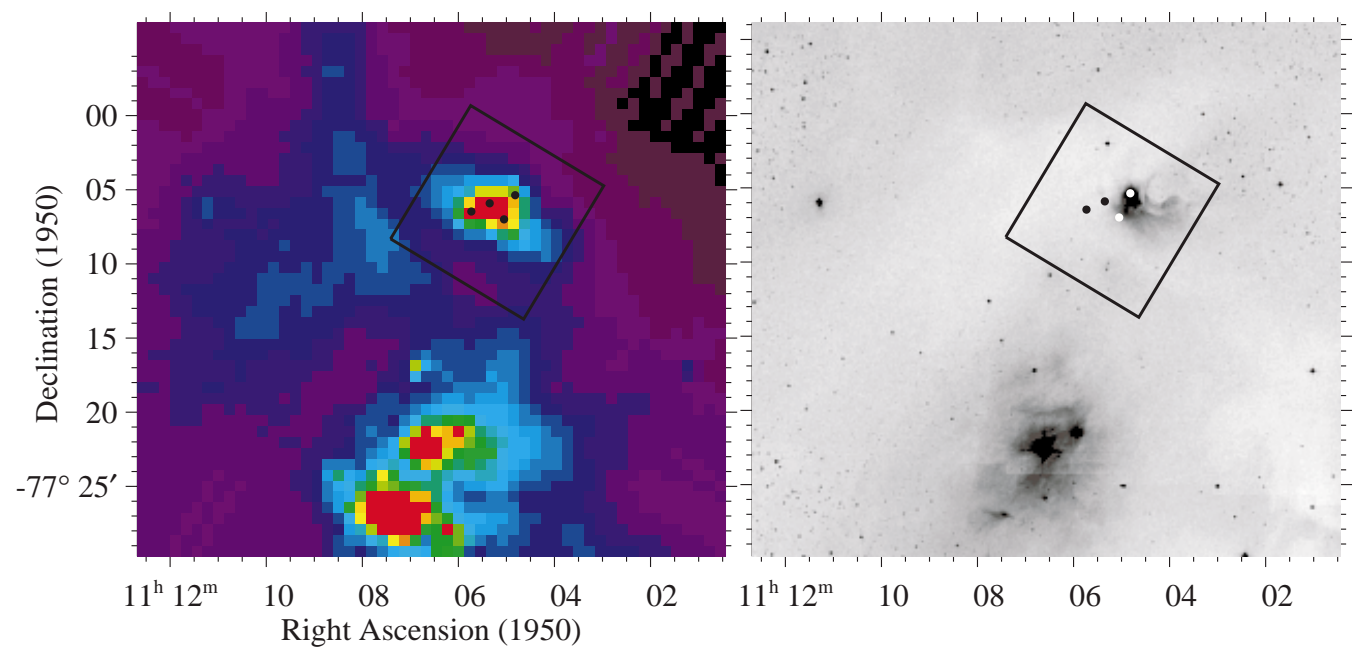

Fig. 1. The ISOPHOT $100 \mu \mathrm{m}$ surface brightness image (left) and $B$-band optical Digitized Sky Survey image (right) of the Chamaeleon I region. The area shown in Fig. 2 is marked with a rectangle, and the sources IRS 2, 4, 6 and Cha-MMS1 are marked with black or white dots. For source identification see Fig. 2
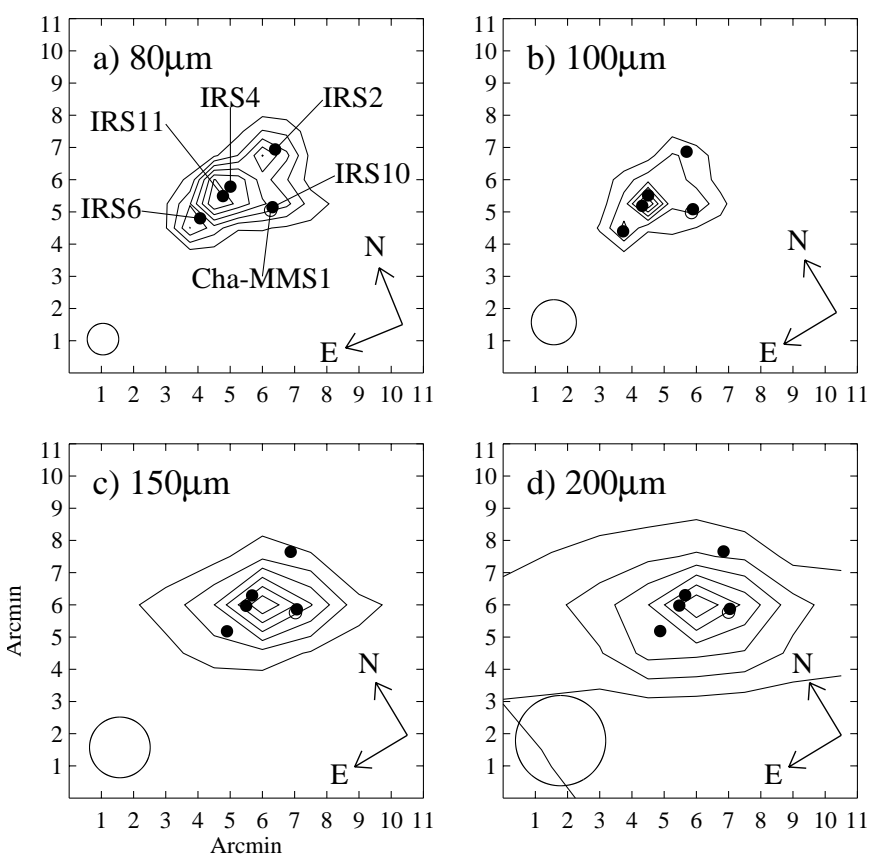

Fig. 2. Contour maps of the Cederblad 110 region at 80 a), 100 b), 150 c) and $200 \mu \mathrm{m} \mathrm{d}$ ). The maps have been deconvolved using a maximum entropy algorithm in the PIA. The filled circles mark the fitted positions of IRS 2, 4, 6, 10 and 11 in the $80 \mu \mathrm{m}$ map. The open circle marks the position of the mm-continuum source Cha-MMS1a as given by Reipurth et al. (1996). The circle at lower left corner shows the size of the Airy disk at each wavelength

and there is only one emission maximum, located approximately at the position of IRS 4. As seen in Fig. 2, the source located south of IRS 2 is close to the position of the mm-continuum source Cha-MMS1. We designate this source, detected in the $80-200 \mu \mathrm{m}$ wavelength range, as Ced 110 IRS 10.
After removing all the known point sources (IRS 2, 4, 6 and Cha-MMS1) and the extended emission from the maps (see Sect. 3.2 below), we find that there is a pointlike or slightly extended emission peak in the residual map at $80 \mu \mathrm{m}$, located between IRS 4 and 6 , as seen in Fig. 3 . The peak is significantly higher than the noise in the residual map. We designate this source as IRS 11. The derived flux density of this source is about $3.5 \mathrm{Jy}$. IRS 11 is not detected at wavelengths longer than $80 \mu \mathrm{m}$ due to confusion. Consequently, the fluxes of IRS 4 may be affected by IRS 11.

\subsection{Determination of flux densities}

The flux densities of the point sources IRS 2, 4, 6 and Cha-MMS1 were determined by fitting the maps with a model consisting of four point sources plus an inclined plane and one (at 80 and $100 \mu \mathrm{m}$ ) or two (at 150 and $200 \mu \mathrm{m}$ ) two-dimensional Gaussian surfaces describing the emission from the extended underlying ridge. The profile of a point source is described by the instrumental footprint as given in PIA. The fitting was performed by using a least-squares minimization routine.

We assume that the far-IR emission of IRS 2, 4 and 6 is located symmetrically around their near-IR positions, given by Cambrésy et al. (1998). Thus we keep their relative positions fixed during the model fitting, but allow the whole triplet to move by maximally $10^{\prime \prime}$. In this way, we allow some positional inaccuracy due to e.g. possible pointing errors of the ISO. In the case of IRS 10 the position is first determined by fitting the $80 \mu \mathrm{m}$ map with a model consisting of the point sources IRS 2, 4, and 6 and the extended ridge, by allowing the position of the IRS 10 to move freely. In subsequent fits at other wavelengths we use this $80 \mu \mathrm{m}$ position but allow $10^{\prime \prime}$ positional uncertainty. 

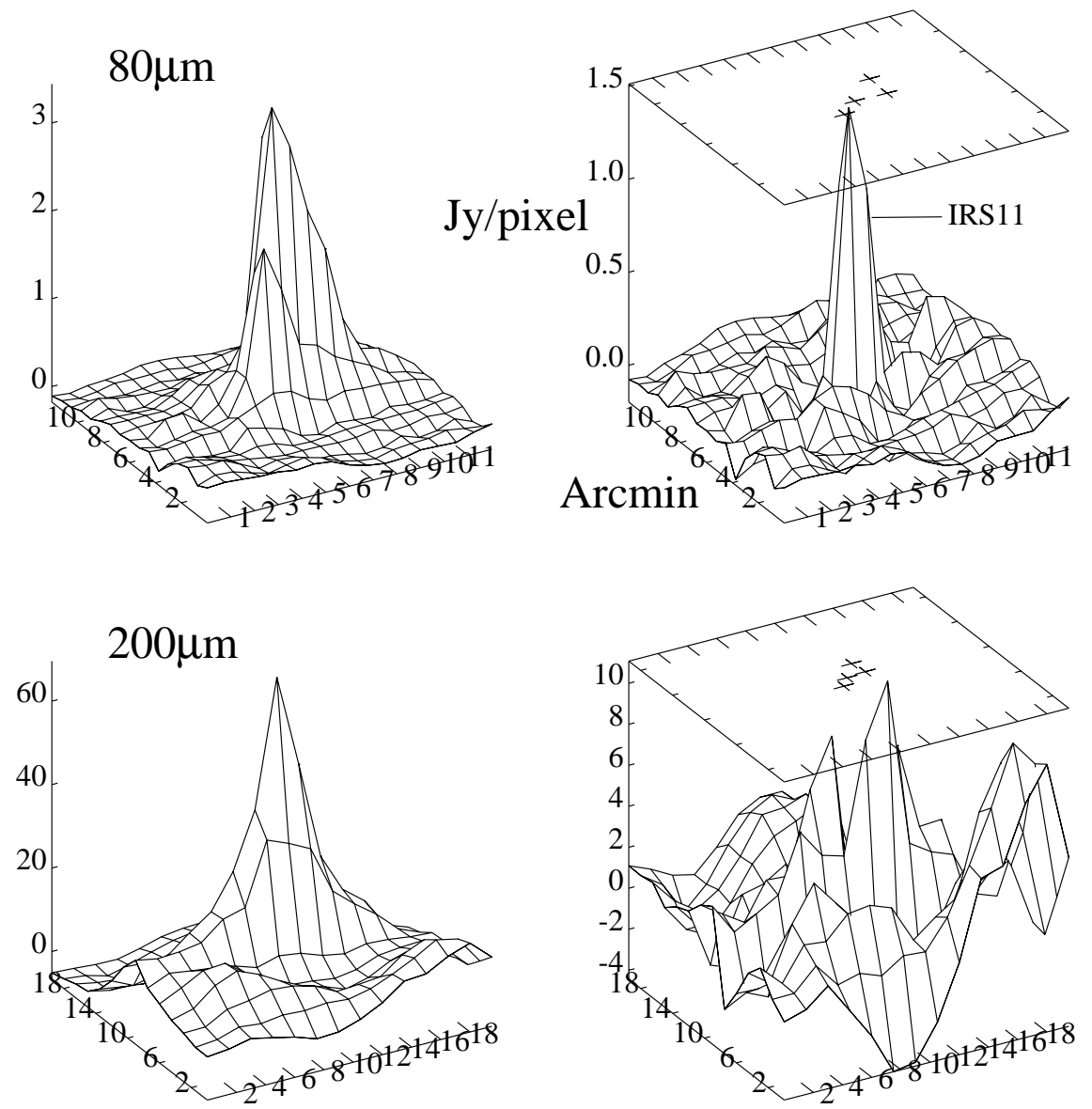

Fig. 3. The observed intensity distributions in Cederblad 110 (left panels) and the residuals after subtraction of the four point sources IRS 2, 4, 6 and 10 and the extended ridge emission (right panels) at 80 and $200 \mu \mathrm{m}$. The $x$ and $y$-axes of the surface plots are in units of arcmins, the $z$-axis is in units of Jy per pixel. The crosses mark the positions of the fitted sources IRS 2 , 4, 6 and 10. The tentative new source IRS 11 as seen at $80 \mu \mathrm{m}$ is indicated with a pointer in the residual map

Table 1. Positions and non-colour corrected flux densities measured with ISOPHOT of compact sources in Cederblad 110. The error values for the flux densities are formal 1- $\sigma$ errors given by the fitting routine, assuming that each pixel has a $10 \%$ uncertainty. ISOPHOT absolute calibration accuracy is 10-15\%. For IRS 2 at $200 \mu \mathrm{m}$ the $2-\sigma$ upper limit is given

\begin{tabular}{|c|c|c|c|c|c|c|c|}
\hline \multirow[t]{2}{*}{ Name } & \multicolumn{2}{|c|}{ Position } & \multirow{2}{*}{$\begin{array}{r}F(80 \mu \mathrm{m}) \\
{[\mathrm{Jy}]}\end{array}$} & \multirow{2}{*}{$\begin{array}{r}F(100 \mu \mathrm{m}) \\
{[\mathrm{Jy}]}\end{array}$} & \multirow{2}{*}{$\begin{array}{r}F(150 \mu \mathrm{m}) \\
{[\mathrm{Jy}]}\end{array}$} & \multirow{2}{*}{$\begin{array}{r}F(200 \mu \mathrm{m}) \\
{[\mathrm{Jy}]}\end{array}$} & \multirow[t]{2}{*}{ Remarks } \\
\hline & $\alpha(1950)$ & $\delta(1950)$ & & & & & \\
\hline Ced 110 IRS 2 & $11^{\mathrm{h}} 4^{\mathrm{m}} 52^{\mathrm{s}} .3$ & $-77^{\circ} 5^{\prime} 36^{\prime \prime}$ & $4.0 \pm 0.6$ & $5.4 \pm 1.0$ & $10.9 \pm 5.6$ & $<9.3$ & \\
\hline Ced 110 IRS 4 & $11^{\mathrm{h}} 5^{\mathrm{m}} 23^{\mathrm{s}} .1$ & $-77^{\circ} 6^{\prime} 09^{\prime \prime}$ & $5.2 \pm 0.8$ & $14.9 \pm 1.7$ & $55.7 \pm 9.9$ & $45.0 \pm 12.1$ & \\
\hline Ced 110 IRS 6 & $11^{\mathrm{h}} 5^{\mathrm{m}} 45^{\mathrm{s}} \cdot 3$ & $-77^{\circ} 6^{\prime} 43^{\prime \prime}$ & $5.1 \pm 0.7$ & $6.1 \pm 1.0$ & $18.2 \pm 6.6$ & $28.3 \pm 10.7$ & \\
\hline Ced 110 IRS 10 & $11^{\mathrm{h}} 5^{\mathrm{m}} 05^{\mathrm{s}} \cdot 6$ & $-77^{\circ} 7^{\prime} 14^{\prime \prime}$ & $2.2 \pm 0.6$ & $5.3 \pm 1.0$ & $31.0 \pm 7.3$ & $31.9 \pm 7.7$ & $=$ Cha-MMS1 \\
\hline Ced 110 IRS 11 & $11^{\mathrm{h}} 5^{\mathrm{m}} 28^{\mathrm{s}} .9$ & $-77^{\circ} 6^{\prime} 20^{\prime \prime}$ & $3.5 \pm 0.8$ & - & - & - & tentative detection $^{1}$ \\
\hline
\end{tabular}

${ }^{1}$ Identified with ISO-ChaI No. 86 (Persi et al. 2000).

The fitted position of IRS 10 is within $\sim 5^{\prime \prime}$ of the position of the mm-continuum source Cha-MMS1 of Reipurth et al. (1996), and thus we propose to identify IRS 10 with Cha-MMS1.

Close to the source IRS 11, at a distance of $18^{\prime \prime}$, Persi et al. (2000) have detected a point source in their 6.7 and $14.3 \mu \mathrm{m}$ ISOCAM survey of the Chamaeleon I cloud (their source ISO-ChaI 86). Within errors these two sources are coincident, and thus we have identified IRS 11 with the source of Persi et al. At the position of the IRS 11, no source has been detected in the DENIS survey (Cambrésy et al. 1998).

Because the point sources are confused, especially in the 150 and $200 \mu \mathrm{m}$ maps, we must verify the existence of each of them before accepting the flux density values given by our model fit. We have made the verification in 


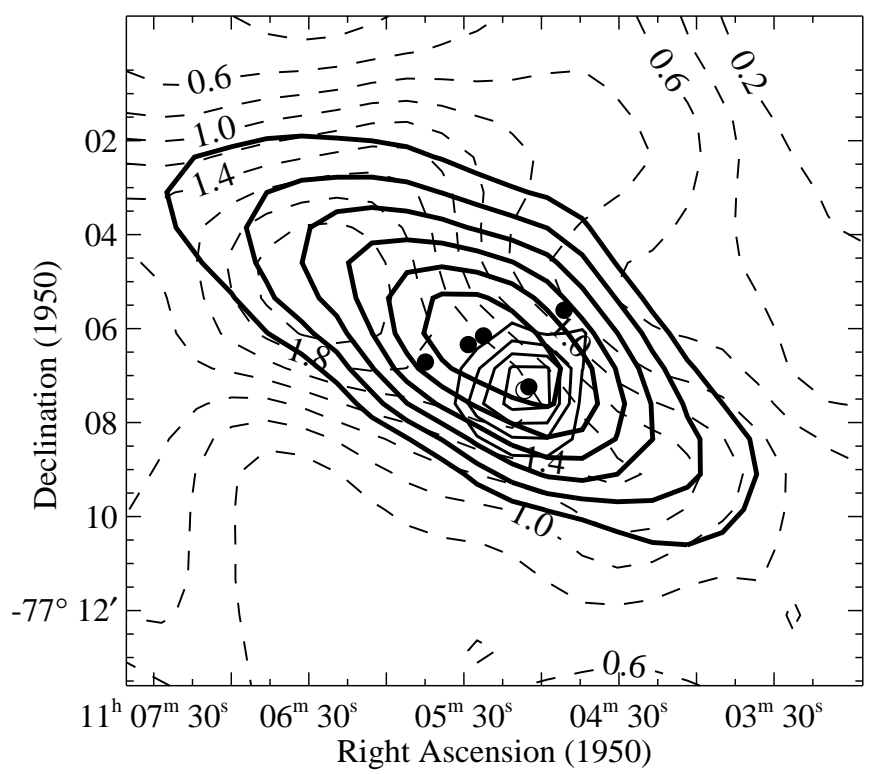

Fig. 4. Contours of $200 \mu \mathrm{m}$ surface brightness of the ridge emission (thick lines). Ridge emission is the emission after subtracting all the fitted point sources. The contours of $200 \mu \mathrm{m}$ surface brightness are from 40 to 115 in steps of $15 \mathrm{MJy} \mathrm{sr}^{-1}$. The fitted positions of the point sources are indicated as in Fig. 2. For comparison the integrated intensities of $\mathrm{HN}^{13} \mathrm{C}$ $(J=1-0)$ (thin lines; the maximum is located near IRS 10) and $\mathrm{C}^{18} \mathrm{O}(J=1-0)$ (dashed lines) emission are shown. The contours of $\mathrm{HN}^{13} \mathrm{C}$ are from 0.1 to 0.4 in steps of $0.1 \mathrm{~K} \mathrm{~km} \mathrm{~s}^{-1}$. The contours of $\mathrm{C}^{18} \mathrm{O}$ are from 0.2 to 2.0 in steps of $0.2 \mathrm{~K} \mathrm{~km} \mathrm{~s}^{-1}$

the following way: We fit the model by forcing each one of the four point sources, one at a time, to have a zero flux density. Then we examine the residuals of the fits and check whether a point source exists at the expected position of the omitted source, having a flux density significantly above the level of the random noise. In this way, we found that each of the sources can be detected at the four wavelengths, except IRS 2 which is not detected at $200 \mu \mathrm{m}$, and IRS 11 , which is only detected at $80 \mu \mathrm{m}$.

In Fig. 3 we show the observed maps and the residuals after the fits at 80 and $200 \mu \mathrm{m}$. The fitted positions (the positions in the $80 \mu \mathrm{m}$ map) and flux densities are listed in Table 1. The errors quoted are formal 1- $\sigma$ errors given by the fitting routine, based on the assumption that each pixel has a $10 \%$ uncertainty. ISOPHOT calibration accuracy for point sources observed in P22 mode has been estimated to be 10-15\% (Klaas et al. 2000). For IRS 2 the flux density given at $200 \mu \mathrm{m}$ is a $2-\sigma$ upper limit.

\subsection{Dense ridge of cold dust}

The emission in the 150 and $200 \mu \mathrm{m}$ maps shows a flattened structure with the main axis in the NE to SW direction. The ridge is much more extended than can be accounted for by co-added emission from the point sources embedded in it. The extended emission is coming from cold dust related to a ridge of dense gas, as seen in the $\mathrm{C}^{18} \mathrm{O}$ maps (Mattila et al. 1989). The region around IRS 10 has been mapped with several high density gas tracers, such as $\mathrm{HNC}(J=1-0), \mathrm{HN}^{13} \mathrm{C}(J=1-0)$ (Harju et al. 1999, private communication) and $\mathrm{HC}_{3} \mathrm{~N}$ ( $J=10-9)$ (Kontinen et al. 2000). These unpublished molecular line maps show a rather circular clump with a diameter of about $2^{\prime}$. Figure 4 shows the distribution of $\mathrm{HN}^{13} \mathrm{C}(J=1-0)$ emission, which is assumed to be optically thin, together with the $200 \mu \mathrm{m}$ surface brightness map of the ridge emission, i.e. emission after subtracting the fitted point sources. The sources IRS 2, 4 and 6 are located just outside the $\mathrm{HN}^{13} \mathrm{C}$ clump, while IRS 10 is located within the clump. The positional coincidence suggests that IRS $10=$ Cha-MMS1 has recently been born in this dense molecular clump.

Integrated intensity of $\mathrm{C}^{18} \mathrm{O}(J=1-0)$ emission (Haikala et al. private communication) is shown in Fig. 4 as dashed contours. There are two clumps which can be separated both spatially and in velocity; one of the clumps is coincident with IRS 10, while the other is located at the north-eastern end of the $200 \mu \mathrm{m}$ ridge, clearly separated from the $\mathrm{HN}^{13} \mathrm{C}(J=1-0)$ clump.

The 150 and $200 \mu \mathrm{m}$ surface brightness values give a colour temperature of about $13 \mathrm{~K}$ (for emissivity $\propto \nu^{2}$ ) at the center of the ridge. Given the temperature and $200 \mu \mathrm{m}$ surface brightness, we can derive the total mass of dust in the ridge inside the lowest surface brightness contour in Fig. 4.

Using Eq. (2) in a form which utilizes the absorption cross section per H-atom $\left(\sigma_{\lambda}^{\mathrm{H}}\right)$ (Hildebrand 1983), we obtain a total mass of $15 M_{\odot}$ by using $\sigma_{200 \mu \mathrm{m}}^{\mathrm{H}}=$ $2.510^{-25} \mathrm{~cm}^{2} \mathrm{H}$-atom ${ }^{-1}$ (Lehtinen et al. 1998).

\section{Discussion}

\subsection{The spectral energy distributions}

We have combined our flux density values with data from Rydgren (1980) (optical flux densities for IRS2), Prusti et al. (1991) (near-IR data for IRS 2, 4 and 6), Prusti et al. (1992) $\left(N_{1}\right.$-band data for IRS 2), Reipurth et al. (1996) (1.3 mm data for Cha-MMS1), Reipurth et al. (1993) (1.3 mm data for IRS 4), Henning et al. (1993) $(1.3 \mathrm{~mm}$ data for IRS 2, 4 and 6), and Persi et al. (2000) (6.7 and $14.3 \mu \mathrm{m}$ data for IRS 11). We note that the $1.3 \mathrm{~mm}$ flux densities of IRS 4 given by Reipurth et al. (1993) and Henning et al. (1993) differ by a factor of about two. We have used the mean value of these fluxes. The resulting SEDs are shown in Fig. 5. We do not use the IRAS CPC values at $50 \mu \mathrm{m}$ because of their calibration uncertainties (Prusti et al. 1991).

All of the sources, except Cha-MMS1, have a SED which is clearly wider than a single-temperature blackbody function. A thorough discussion of the SEDs would require detailed modelling of the spatial distribution of the circumstellar dust, possibly in a disk, which is beyond the scope of this work. However, we can expect that at $\sim 80-200 \mu \mathrm{m}$, and at $1.3 \mathrm{~mm}$ when available, the emission is coming from "classical" big grains, which are at an 

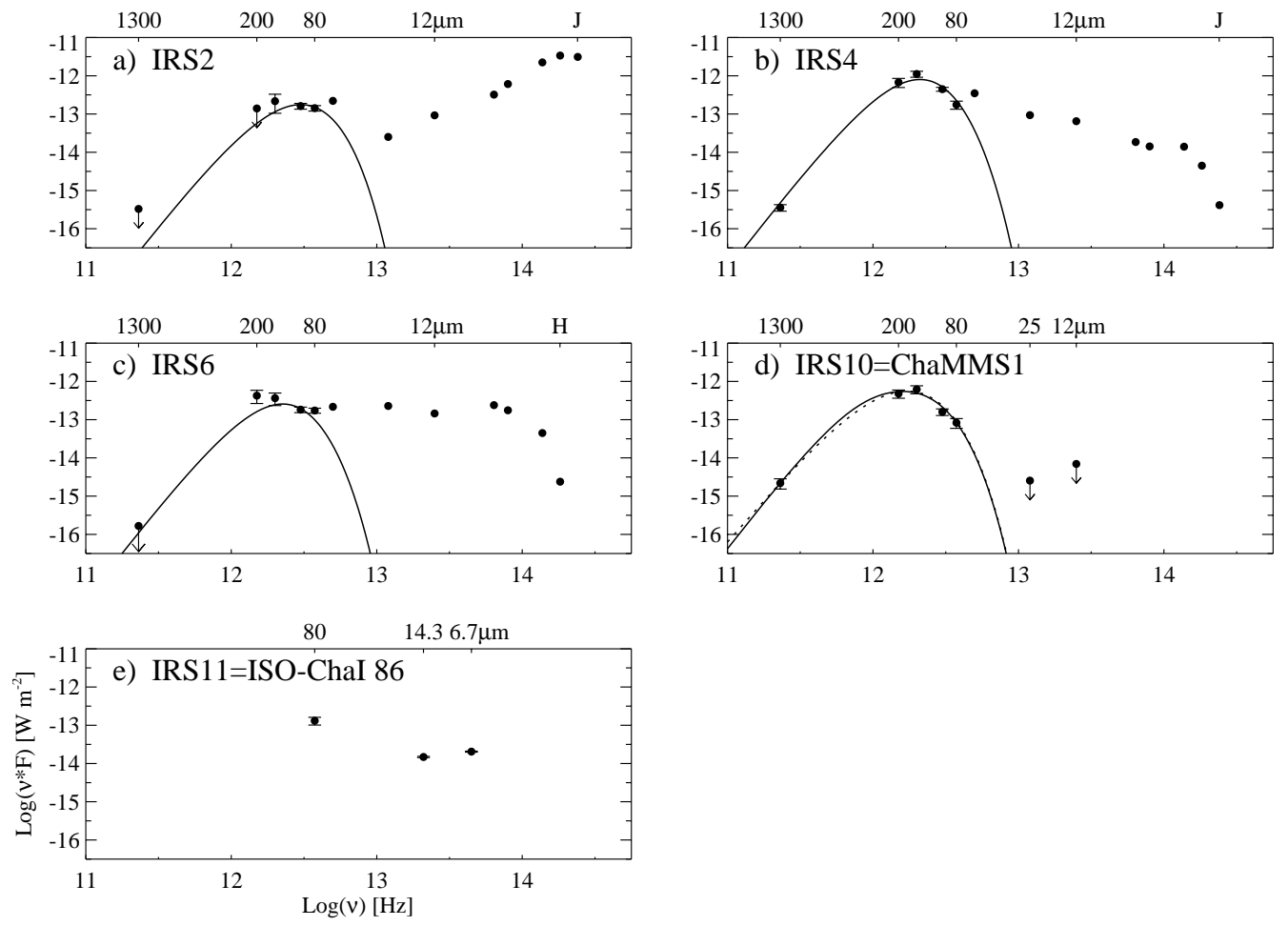

Fig. 5. The spectral energy distributions and modified blackbody fits with emissivity index $\beta=2.0$ for the sources IRS 2 a), IRS 4 b), IRS $6 \mathbf{c}$ ), and IRS $10=$ Cha-MMS1 d). The flux densities of IRS 6 at $\lambda>80 \mu$ m can not be fitted with a single blackbody (see text); the fit shown is merely an example of a blackbody with $T_{\mathrm{d}}=22 \mathrm{~K}$. For IRS 10 we show two fits: the solid line is a fit with $\beta=2.0$, and the dotted line is with $\beta=1.5$. The $1-\sigma$ error bars are given. The available data for IRS 11 are shown in e)

Table 2. The fitted parameters for sources in Cederblad 110. $\beta$ is the power law index of the dust opacity. $T_{\mathrm{d}}$ is the derived dust temperature. $\lambda_{0}$ is the wavelength (corresponding to $\nu_{0}$ ) for which the optical depth is unity. The sub-mm luminosity $L_{\mathrm{sub}-\mathrm{mm}}$ is the luminosity of the fitted blackbody function, integrated longward of $350 \mu \mathrm{m}$. $L_{\mathrm{bol}}$ is the bolometric luminosity and $T_{\mathrm{bol}}$ the bolometric temperature which are derived as described in the text. $M_{\mathrm{cs}}^{200 \mu \mathrm{m}}$ and $M_{\mathrm{cs}}^{1.3 \mathrm{~mm}}$ are the circumstellar masses derived by using the $200 \mu \mathrm{m}$ and $1.3 \mathrm{~mm}$ fluxes, respectively. "Relative $M_{\mathrm{cs}}$ " is the relative circumstellar mass with respect to the mass of IRS4. The last column lists the YSO class (see text). The parameters for IRS 6 are mean values in the 18-24 K dust temperature range because its far-IR/sub-mm SED cannot be fitted with a single blackbody (see text)

\begin{tabular}{|c|c|c|c|c|c|c|c|c|c|c|c|}
\hline Name & $\beta$ & $\begin{array}{r}T_{\mathrm{d}} \\
{[\mathrm{K}]}\end{array}$ & $\begin{array}{r}\lambda_{0} \\
{[\mu \mathrm{m}]}\end{array}$ & $\begin{array}{r}L_{\mathrm{sub}-\mathrm{mm}} \\
{\left[L_{\odot}\right]}\end{array}$ & $\begin{array}{c}L_{\mathrm{bol}} \\
{\left[L_{\odot}\right]}\end{array}$ & $\frac{L_{\mathrm{sub}-\mathrm{mm}}}{L_{\mathrm{bol}}}$ & $\begin{array}{r}T_{\mathrm{bol}} \\
{[\mathrm{K}]}\end{array}$ & Relative $M_{\mathrm{cs}}$ & $\begin{array}{r}M_{\mathrm{cs}}^{200 \mu \mathrm{m}} \\
\quad\left[M_{\odot}\right]\end{array}$ & $\begin{array}{l}M_{\mathrm{cs}}^{1.3 \mathrm{~mm}} \\
\quad\left[M_{\odot}\right]\end{array}$ & YSO Class \\
\hline Ced 110 IRS2 & 2 & 25 & 2 & 0.001 & 3.4 & $3 \mathrm{E}-4$ & 2700 & - & 0.006 & - & II-III \\
\hline Ced 110 IRS4 & 2 & 21 & 158 & 0.02 & 1.0 & 0.02 & 72 & 1.0 & 0.16 & 0.14 & I \\
\hline Ced 110 IRS6 & 2 & $18-24$ & - & $\sim 0.008$ & $\sim 0.8$ & $\sim 0.01$ & $\sim 260$ & - & $\sim 0.04$ & - & I \\
\hline Ced 110 IRS10 & 2 & 20 & 509 & 0.06 & 0.46 & 0.1 & 20 & 5.0 & - & 0.45 & 0 \\
\hline Ced 110 IRS10 & 1.5 & 20 & 350 & 0.04 & 0.44 & 0.1 & 20 & 5.1 & - & 0.45 & 0 \\
\hline
\end{tabular}

equilibrium temperature with the radiation field. Thus we have fitted the flux densities at these wavelengths with a modified blackbody of the form

$F_{\nu}=B_{\nu}\left(T_{\mathrm{d}}\right)\left(1-\exp \left(-\tau_{\nu}\right)\right) \Omega_{\mathrm{s}}$

where $B_{\nu}\left(T_{\mathrm{d}}\right)$ is the Planck function at the dust temperature $T_{\mathrm{d}}, \tau_{\nu}$ is the optical depth which is assumed to vary with frequency as $\tau_{\nu}=\left(\nu / \nu_{0}\right)^{\beta}, \Omega_{\mathrm{s}}$ is the solid angle of the emitting reqion, and $\nu_{0}$ is the frequency where the optical depth is unity. We have assumed that the emissivity law index $\beta$ is 2 . In the case of IRS 10 , we have tried values of $\beta$ between 1 and 2 , and found out that for values
$<1.5$ the SED cannot be fitted with physically meaningful parameters.

The fitted blackbody functions are shown in Fig. 5, and the resulting temperatures and other parameters are listed in Table 2. The ISO flux densities, which are at the peak of the SEDs, mainly set the temperature of the fitted blackbodies, while the $1.3 \mathrm{~mm}$ flux densities help to determine the value of $\nu_{0}$.

In the case of IRS 6 we cannot fit all the four ISO flux densities with a blackbody which is consistent with the $1.3 \mathrm{~mm}$ flux density upper limit. By making the fit by trial and error we find out that the 100 and $150 \mu \mathrm{m}$ flux 
densities can be fitted with a blackbody with a temperature in the range $18-24 \mathrm{~K}$, while still having reasonable values for $\nu_{0}$ and $\Omega_{\mathrm{s}}$ and a $1.3 \mathrm{~mm}$ flux density which is less than or equal to the observed upper limit. With these parameters also the ISO 80 and $200 \mu \mathrm{m}$ flux densities agree within $2-\sigma$ with the fits. The parameters for IRS 6 listed in Table 2 are estimated mean values in the $18-24 \mathrm{~K}$ temperature range.

In the case of IRS 2, 4 and 10, the four ISO flux densities at $80-200 \mu \mathrm{m}$ and the millimeter continuum flux densities (when available), are well fitted with a single modified blackbody emission curve. In the case of IRS 6 , the $80 \mu \mathrm{m}$ flux density is already higher than the fitted blackbody curve. At $\lambda<60-80 \mu \mathrm{m}$, emission from small grains or from hotter large grains starts to dominate the SED for IRS 2 and 4.

\subsection{The luminosities and masses}

We have derived bolometric luminosities for all sources by summation of the observed flux density values, and extrapolation to infinite wavelengths on the longward side. We have neglected the luminosity shortward of the observed range, except for Cha-MMS1. According to Prusti et al. (1992), this omitted luminosity is $\leq 10 \%$ of the total luminosity for these sources. The derived bolometric luminosities, $L_{\mathrm{bol}}$, are listed in Table 2 , and are similar to those derived by Prusti et al. (1991).

The fitted values of $\lambda_{0}$, i.e. the wavelength where $\tau_{\text {dust }}=1$ (see Table 2), suggest that for IRS 2, the $200 \mu \mathrm{m}$ emission is optically thin, for IRS 4 and 6 moderately optically thin, while for IRS 10 it is optically thick. Thus, we can estimate the total (gas plus dust) circumstellar mass, $M_{\mathrm{cS}}$, using the fitted $200 \mu \mathrm{m}$ (IRS 2, 4 and 6) or $1300 \mu \mathrm{m}$ (IRS 4 and 10) flux density and the derived dust temperature. In order to correct for the non-vanishing optical depths of IRS 4 and 6 at $200 \mu \mathrm{m}$, we have applied a correction factor of $[\tau(200 \mu \mathrm{m}) /(1-\exp (-\tau(200 \mu \mathrm{m})))]=2.0$ and 1.8 , to the respective observed flux densities.

In order to estimate the mass of circumstellar matter $M_{\mathrm{cs}}$ associated with the cold dust we use the equation

$M_{\mathrm{cs}}=\frac{F_{\nu} D^{2}}{R \kappa_{\nu} B_{\nu}\left(T_{\mathrm{d}}\right)}$

with $D$ the distance, $R$ the dust-to-gas mass ratio and $\kappa_{\nu}$ the mass absorption coefficient of the assumed isothermal dust (see e.g. Hildebrand 1983; Chini et al. 1987).

Although the absolute values of $M_{\mathrm{cs}}$ derived from Eq. (2) are quite uncertain, mostly due to the uncertain values of $\kappa_{\nu}$ and $R$, the relative values of $M_{\mathrm{cs}}$ are much more reliable because they do not require the knowledge of $D, R$ or $\kappa_{\nu}$.

The absolute values of masses in Table 2 have been estimated by adopting $R=1 / 100$ and $\kappa_{1300 \mu \mathrm{m}}=1 \mathrm{~cm}^{2} \mathrm{~g}^{-1}$ (Ossenkopf \& Henning 1995). The value of $\kappa_{200 \mu \mathrm{m}}$ has been scaled from $\kappa_{1300 \mu \mathrm{m}}$ by using a $\lambda^{-2}$ dependence.

Based on the relative circumstellar masses of IRS 10 and 4 , the amount of circumstellar mass decreases by a factor of about five from YSO Class 0 to Class I, which is similar to the factor of 5-10 found by André \& Montmerle (1994).

\subsection{The classification of IRS 2, 4 and 6}

Young stellar objects have been classified into evolutionary classes based on the spectral index between two wavelengths, e.g. 2.2 and $25 \mu \mathrm{m}$ (e.g. Wilking et al. 1989). However, this method uses only two flux density values of the whole SED. From Fig. 5 one can see that although the sources IRS 4 and 6 both belong to the same class when using this near-IR spectral index, their SEDs are quite different. Clearly we need a classification criterion which uses the whole SED.

Myers \& Ladd (1993) have introduced the bolometric temperature $\left(T_{\mathrm{bol}}\right)$ as a measure of the evolutionary status of a YSO. This method uses all the information available in the SED, and can be applied to a SED observed over any wavelength range as long as the SED is sufficiently well covered (especially around its maximum). According to Chen et al. (1995), the division values of $T_{\text {bol }}$ between classes 0 , I, II and III are 70, 650 and $2800 \mathrm{~K}$, respectively.

To derive $T_{\mathrm{bol}}$ one has to calculate the first and zeroth $\left(=L_{\mathrm{bol}}\right)$ moments of the SED. The integral for the $1 \mathrm{st}$ moment over the SED has been evaluated in a way similar to that used by us to calculate the bolometric luminosity. The derived values of $T_{\mathrm{bol}}$ are $2700,72,260$ and $19 \mathrm{~K}$ for IRS 2, 4, 6 and 10, respectively. Following the classification of Chen et al. (1995), these sources belong to classes III, I, I and 0, respectively.

Chen et al. (1995) have discussed the error estimation of the derived $T_{\mathrm{bol}}$ and $L_{\mathrm{bol}}$ values. They concluded that the overall uncertainty factor of $T_{\mathrm{bol}}$ is about 1.5 for embedded sources (IRS 4, 6 and 10 in our case), and about 2 for T Tauri stars (IRS 2). $T_{\text {bol }}$ of IRS 2 is close to the division value of classes II and III, and thus we cannot definitely decide, whether IRS 2 belongs to class II or III purely by the value of $T_{\text {bol }}$. $T_{\text {bol }}$ of IRS 4 is much lower than that of IRS 6 , since its SED rises much more steeply towards the far-IR than that of IRS 6 . The value of $T_{\mathrm{bol}}$, $72 \mathrm{~K}$, is only slightly higher than the division value between classes 0 and I and, within errors, IRS 4 could be classified as a Class 0 object as well. However, IRS 4 does not fulfil the other characteristics of a Class 0 object (see the criteria listed in Sect. 4.4 below), and thus we classify it as a Class I source.

\subsection{The evolutionary status of IRS $10=$ Cha-MMS1}

As suggested by Reipurth et al. (1996), IRS 10 may be a real protostar. Such a Class 0 object has to fulfil the following criteria (André et al. 1993): 1) non-detectability at wavelengths shorter than about $10 \mu \mathrm{m}, 2)$ the SED is well characterized by a single modified blackbody with a temperature of about $15-30 \mathrm{~K}, 3$ ) there exists indirect evidence for the presence of a central protostellar object 
(e.g. a molecular outflow or a cm-wavelength continuum source), and 4) the ratio $L_{\mathrm{sub}-\mathrm{mm}} / L_{\mathrm{bol}}>510^{-3}$, where $L_{\text {sub-mm }}$ is the luminosity for wavelengths greater than $350 \mu \mathrm{m}$.

In order to check the validity of criterion \#1, we have obtained HIRES processed IRAS images, provided by IPAC ${ }^{3}$. In the 12 and $25 \mu \mathrm{m}$ images the resolution is adequate to resolve all four components because IRS 2, 4 and 6 are clearly resolved. However, IRS 10 is not detected at either wavelength. We have estimated 2- $\sigma$ upper limits of 0.03 and $0.02 \mathrm{Jy}$ for IRS 10 at 12 and $25 \mu \mathrm{m}$ from the HIRES maps. Cha-MMS1 is also not detected in the DENIS near-IR survey with limiting magnitudes of 18,16 and 13.5 in $I, J$ and $K_{\mathrm{s}}$ bands, respectively (Cambrésy et al. 1998).

Based on the available flux density values between 80 $1300 \mu \mathrm{m}$, condition \#2 is fulfilled. Condition \#3 is fulfilled if we assume that the $\mathrm{CO}$ outflow is driven by IRS 10 , but this should be verified with higher angular resolution observations. Most of the known Class 0 objects have been detected by their cm-continuum observations, and such observations would be very instructive in this case.

For IRS $10 L_{\mathrm{sub}-\mathrm{mm}} / L_{\mathrm{bol}} \approx 0.1$ (see Table 2 ). Thus, condition \#4 is also fulfilled. Based on the currently available data, we conclude that IRS 10 meets all the criteria of a Class 0 object.

André (1999) has compiled a list of confirmed Class 0 protostars. The mean values, together with minimum and maximum values in parentheses, of $L_{\mathrm{bol}}, M_{\mathrm{cs}}$, $L_{\text {sub-mm }} / L_{\text {bol }}$, and $T_{\text {bol }}$ are $12(0.15,75) L_{\odot}, 2.1$ $(0.5,7) M_{\odot}, 4.2(2,12) \%$ and $44(18,70) \mathrm{K}$ (excluding the massive Class 0 objects). IRS 10 is at the low end of $L_{\mathrm{bol}}, M_{\mathrm{cs}}, T_{\mathrm{bol}}$, and at the high end of $L_{\mathrm{sub}-\mathrm{mm}} / L_{\mathrm{bol}}$ distribution of the observed Class 0 objects.

A protostar derives a substantial fraction of its luminosity from accretion. The accretion luminosity is $L_{\mathrm{acc}}=$ $\left(G M_{\star} \dot{M}\right) / R_{\star}$, where $M_{\star}$ is the protostellar mass, $\dot{M}$ is the mass accretion rate, and $R_{\star}$ is the radius of the protostar onto which the material is accreting. If IRS 10 is an accreting Class 0 protostar, its protostellar mass is less than its circumstellar mass, i.e. $M_{\star}<0.46 M_{\odot}$. If we use $R_{\star}=$ $3 R_{\odot}$, we obtain an accretion rate $\dot{M}>10^{-7} M_{\odot} \mathrm{yr}^{-1}$. The required accretion rate does not conflict with the expected rate for Class 0 objects, $10^{-5}-10^{-6} M_{\odot} \mathrm{yr}^{-1}$.

\subsection{Star formation efficiency and epochs of star formation in Ced 110}

We have estimated the mass of the high column density $\left(N\left(\mathrm{C}^{18} \mathrm{O}\right) \gtrsim 1.210^{15} \mathrm{~cm}^{-2}\right)$ molecular gas clump as delineated by the $\mathrm{C}^{18} \mathrm{O}$ emission (see Fig. 4) to be $19 M_{\odot}$. We included the clump area inside the lowest $200 \mu \mathrm{m}$ surface brightness contour in Fig. 4 (the same area as used for the mass derived from the $150 / 200 \mu \mathrm{m}$ maps), and adopted the $\mathrm{C}^{18} \mathrm{O}$ to $\mathrm{H}_{2}$ conversion factor as determined

\footnotetext{
${ }^{3}$ IPAC is funded by NASA as part of the IRAS extended mission under contract to JPL.
}

by Harjunpää \& Mattila (1996) for Cha I. This value is in good agreement with the mass estimate of $15 M_{\odot}$ derived from the 150/200 $\mu \mathrm{m}$ emission (see Sect. 3.3). We estimate the mass of the stellar objects to be 3 to $4 M_{\odot}$ whereas we have adopted a mass of $1 M_{\odot}$ for IRS 2 , and for the four other objects masses of 0.5 to $0.75 M_{\odot}$. Under the assumption that all these objects have formed from the available material in the Cederblad 110 dense core, we end up with a star formation efficiency of 16 to $21 \%$. This is considerably higher than the value of 2 to $9 \%$ in the Taurus cloud (Cohen \& Kuhi 1979) and is only slightly lower than the value of $\gtrsim 22 \%$ found for the $\rho$ Oph dense core (Wilking et al. 1989). The star formation efficiency found for the Cederblad 112 region in the northern part of Chamaeleon I is even higher, $35 \%$ (Mattila et al. 1989; Oasa et al. 1999).

The five YSOs in Cederblad 110 are within an area of $0.1 \times 0.1 \mathrm{pc}^{2}$ which corresponds to a local surface density of $\sim 500$ stars $\mathrm{pc}^{-2}$. This surface density is comparable to the values of $\sim 280$ and $\sim 210 \mathrm{stars}_{\mathrm{pc}}^{-2}$ found in the $\rho \mathrm{Oph}$ (Comeron et al. 1993) and Cederblad 112 (Oasa et al. 1999) cores, respectively, and it is substantially higher than the value of $\sim 0.3$ stars $\mathrm{pc}^{-2}$ in the Taurus cloud (Cohen \& Kuhi 1979; Herbig \& Bell 1988).

With increasing resolution and sensitivity the single FIR source (Baud 35) associated with Cederblad 110 in Baud et al. (1984) was resolved into three separate objects by Prusti et al. (1991), and now into five sources by us. The detection of the multiplicity of the Cederblad 110 FIR source suggests that many other IRAS FIR sources might be multiple as well, and simply unresolved because of their larger distances.

The visible G type star IRS 2 is located outside the dense clump of gas, in a low-density bay of the molecular gas distribution (see Fig. 4). High dispersion optical spectra (Walter 1992) have confirmed its nature as a PMS star, and have revealed it to be in rapid rotation with $V \sin i \sim$ $75 \mathrm{~km} \mathrm{~s}^{-1}$. While the embedded objects IRS 4, 6, 10, and 11 must have formed very recently, up to $\sim 10^{5}$ years ago, IRS 2 is substantially older ( $\left.\sim 10^{6} \mathrm{yrs}\right)$. Thus, at least two distinct star formation episodes have occurred during the Cederblad 110 history. This indicates that the first generation of stars has not inhibited further star formation, but rather may have induced it.

The association of IRS 2 with a dark core, its being the illuminating star of a reflection nebula, and the presence of $\mathrm{H} \alpha$ emission in its spectrum (Walter 1992) suggest a connection with the class of Herbig Ae/Be stars (HAEBEs). Recent NIR surveys by Testi et al. (1997; 1998; 1999) and Hillenbrand et al. (1995) have shown that HAEBEs are associated with small clusters or groupings of newly-born, low mass stars. In their sample the groups become scarce (2 to 4 members) for HAEBE stars of spectral types later than A5. Our results suggest that even central stars of spectral type later than A can be associated with star formation in small dense groups. Such groups may have escaped detection if they are dissolving faster than the groups near B and A type stars, and are thus 
observable only during the very early phases of stellar formation when the stars are still deeply embedded. Only a few other dense groups of embedded low mass YSOs are known, e.g. in NGC 1333 (NGC 1333-IRAS 2, 4A, 4B, SVS 13B, see Lefloch et al. 1998; Sandell et al. 1994) and in L 1448 (L 1448-IRS2, C, N, see O'Linger et al. 1999).

\section{Conclusions}

We have revisited the Cederblad 110 reflection nebula with ISO's far-IR cameras and mapped it with higher spatial resolution and at longer wavelengths than before.

1. We have detected a new far-IR source previously detected only by its $1.3 \mathrm{~mm}$ continuum emission. It is designated IRS 10 and is classified as a Class 0 protostar;

2. We have detected another far-IR source at $80 \mu \mathrm{m}$ close to IRS 4 and 6 , designated IRS 11 ;

3. Three previously known IR-sources in Cederblad 110 have been measured for the first time at wavelengths longer than $100 \mu \mathrm{m}$ and far-IR SEDs are presented;

4. The sources span a wide range of evolutionary phases, from Class 0 protostar to Class III Weak-line T-Tauri star;

5. At the wavelengths $\lambda \gtrsim 80 \mu \mathrm{m}$ the emission is expected to be dominated by "classical" big grains, which are at equilibrium temperature. This has enabled us to estimate the temperature of the circumstellar cold dust, and to derive the circumstellar mass associated with it. For the sources IRS 2, 4 and 10 the far-infrared emission at wavelengths $\lambda \geq 80 \mu \mathrm{m}$ can be well fitted with a single modified blackbody with dust temperatures of 20-25 K;

6. By combining our data with previous flux density measurements, we have obtained rather complete SEDs for these sources, and have been able to derive bolometric luminosities and temperatures;

7. At all wavelengths $80-200 \mu \mathrm{m}$ we have detected an extended emission ridge. Embedded in it is a clump of dense molecular material. The sources IRS 2, 4, 6 , 10 and 11 are embedded in the ridge, representing the parental material out of which these stars have formed. The Class 0 source IRS 10 is located close to the maximum of a $\mathrm{HN}^{13} \mathrm{C}(J=1-0)$ column density map;

8. The star formation efficiency in the Cederblad 110 dense core area is about $20 \%$.

Acknowledgements. The work of K.L., L.H. and K.M. has been supported by the Academy of Finland through grant No. 1011055. We thank Carlos Gabriel (ISO Data Centre, Villafranca) for providing us with the IDL runtime version of PIA. The least-squares minimization routine used is made available in IDL code by C. B. Markwardt (http://astrog.physics.wisc.edu/ craigm/idl/idl.html), which is acknowledged. We are grateful to J. Harju for letting us use his unpublished molecular line data. We thank P. Persi, A. R. Marenzi and G. Olofsson for permission to quote their ISOCAM data before publication. ISOPHOT and the Data Centre at MPIA, Heidelberg, are funded by the Deutsches Zentrum für Luft- und Raumfahrt DLR and the Max-Planck-Gesellschaft. We thank the referee whose comments improved the article.

\section{References}

Adams, F. C., Lada, C. J., \& Shu, F. H. 1987, ApJ, 312, 788

André, P., Ward-Thompson, D., \& Barsony, M. 1993, ApJ, 406, 122

André, P., \& Montmerle, T. 1994, ApJ, 420, 837

André, P., Ward-Thompson, D., \& Barsony, M. 1999, in Protostars and Planets IV, ed. V. Mannings, A. P. Boss, \& S. S. Russell (Tucson: University of Arizona Press), in press

Baud, B., Beintema, D. A., Wesselius, P. R., et al. 1984, ApJ, 278, L53

Cambrésy, L., Copet, E., \& Epchtein, N. 1998, A\&A, 338, 977

Chen, H., Myers, P. C., Ladd, E. F., et al. 1995, ApJ, 445, 377

Chini, R., Krügel, E., \& Wargau, W. 1987, A\&A, 181, 378

Cederblad, S. 1946, Medd. Lunds. Astron. Obs. Series II, No. 119

Cohen, M., \& Kuhi, L. V. 1979, ApJS, 41, 743

Comeron, F., Rieke, G. H., Burrows, A., et al. 1993, ApJ, 416, 185

Gabriel, C., et al. 1997, The ISOPHOT Interactive Analysis PIA, a calibration and scientific analysis tool, in Astronomical Data Analysis Software and Systems (ADASS) VI, ed. G. Hunt, \& H. E. Payne (San Francisco: ASP), ASP Conf. Ser., 125, 108

Gauvin, L. S., \& Strom, K. M. 1992, ApJ, 385, 217

Gürtler, J., Schreyer, K., \& Henning, Th. 1999, A\&A, 346, 205

Harjunpää, P., \& Mattila, K. 1996, A\&A, 305, 920

Henning, Th., Pfau, W., Zinnecker, H., et al. 1993, A\&A, 276, 129

Herbig, G., \& Bell, K. 1988, Lick Obs. Bull., 1111, 1

Hildebrand, R. H. 1983, QJRAS, 24, 267

Hillenbrand, L. A., Meyer, M. R., Strom, S. E., et al. 1995, AJ, 109,280

Kessler, M. F., Steinz, J. A., \& Anderegg, M. E. 1996, A\&A, $315, \mathrm{~L} 27$

Klaas, U., Laureijs, R. J., Radovich, M., et al. 2000, ISOPHOT Calibration Accuracies, SAI/1998-092/Dc, Version 3.0, January 2000

Kontinen, S., Harju, J., Heikkilä, A., et al. 2000, A\&A, 361, 704

Knude, J., \& Høg, E. 1998, A\&A, 338, 897

Lada, C. J., \& Wilking, B. 1984, ApJ, 287, 610

Lada, C. J. 1987, in IAU Symposium No. 115: Star Forming Regions, ed. M. Peimbert, \& J. Jugaku (Dordrecht Reidel), 1

Lefloch, B, Castets, A., Cernicharo, J., et al. 1998, A\&A, 334, 269

Lehtinen, K., Lemke, D., Mattila, K., et al. 1998, A\&A, 333, 702

Lemke, D., Klaas, U., Abolins, J., et al. 1996, A\&A, 315, L64

Mattila, K., Liljeström, T., \& Toriseva, M. 1989, in ESO workshop on Low Mass Star Formation and Pre-Main Sequence Objects, ed. B. Reipurth (ESO, Garching), 153

Myers, P. C., \& Ladd, E. F. 1993, ApJ, 413, L47

O'Linger, J., Wolf-Chase, G., Barsony, M., et al. 1999, AJ, 515, 696 
Oasa, Y., Tamura, M., \& Sugitani, K. 1999, ApJ, 526, 336

Ossenkopf, V., \& Henning, Th. 1994, A\&A, 291, 943

Persi, P., Marenzi, A. R., Olofsson, G., et al. 2000, A\&A, 357, 219

Prusti, T., Clark, F. O., Whittet, D. C. B., et al. 1991, MNRAS, 251, 303

Prusti, T., Whittet, D. C. B., \& Wesselius, P. 1992, MNRAS, 254,361

Reipurth, B., Chini, R., Krügel, E., et al. 1993, A\&A, 273, 221

Reipurth, B., Nyman, L.-Å., \& Chini, R. 1996, A\&A, 314, 258

Rydgren, A. E. 1980, AJ, 85, 444
Sandell, G., Knee, L. B. G., Aspin, C., et al. 1994, A\&A, 285, L1

Schwartz, R. D. 1991, in Low mass star formation in southern molecular clouds, ed. B. Reipurth, ESO Sc. Report, No. 11, 93

Testi, L., Palla, F., Prusti, T., et al. 1997, A\&A, 320, 159

Testi, L., Palla, F., \& Natta, A. 1998, A\&AS, 133, 81

Testi, L., Palla, F., \& Natta, A. 1999, A\&A, 342, 515

Walter, F. M. 1992, AJ, 104, 758

Wilking, B. A., Lada, C. J., \& Young, E. T. 1989, ApJ, 340, 823 